\title{
Gluing Formula for Casimir Energies ${ }^{\dagger}$
}

\author{
Klaus Kirsten ${ }^{1, *}$ and Yoonweon Lee ${ }^{2}$ \\ Department of Mathematics, Baylor University, Waco, TX 76796, USA \\ 2 Department of Mathematics, Inha University, Incheon 402-751, Korea; yoonweon@inha.ac.kr \\ * Correspondence: klaus_kirsten@baylor.edu \\ + Presented at Symmetry 2017-The First International Conference on Symmetry, Barcelona, Spain, \\ 16-18 October 2017.
}

Published: 3 January 2018

Let $M_{1}$ and $M_{2}$ be two Riemannian manifolds each of which has the boundary $N$. Consider the Laplacian on $M_{1}$ and $M_{2}$ augmented with Dirichlet boundary conditions on $N$. A natural question to ask is whether there is any relation between spectral properties of the Laplacian on $M_{1}, M_{2}$, and the Laplacian on the manifold $M$ (without boundary) obtained by gluing together $M_{1}$ and $M_{2}$, namely $M$ $=M_{1} \cup_{N} M_{2}$. A partial answer is given by the Burghelea-Friedlander-Kappeler-gluing formula for zetadeterminants. This formula contains an (in general) unknown polynomial which is completely determined by some data on a collar neighborhood of the hypersurface $N$. In this talk, I present results for the polynomial in terms of suitable geometric tensors on $N$. Choosing $M_{1}, M_{2}$ and $M$ as appropriate, results in a gluing formula for Casimir energies.

Conflicts of Interest: The authors declare no conflict of interest.

C 2018 by the authors. Licensee MDPI, Basel, Switzerland. This article is an open access article distributed under the terms and conditions of the Creative Commons Attribution (CC BY) license (http://creativecommons.org/licenses/by/4.0/). 